\title{
Electronic and vibrational contributions to first hyperpolarizability of donor-acceptor-substituted azobenzene
}

\author{
Robert Zaleśny, ${ }^{1, a)}$ Ireneusz W. Bulik, ${ }^{1, b)}$ Wojciech Bartkowiak, ${ }^{1}$ Josep M. Luis, ${ }^{2}$ \\ Aggelos Avramopoulos, ${ }^{3}$ Manthos G. Papadopoulos, ${ }^{3}$ and Przemysław Krawczyk ${ }^{4}$ \\ ${ }^{1}$ Theoretical Chemistry Group, Institute of Physical and Theoretical Chemistry, Wroctaw University of \\ Technology, Wyb. Wyspiańskiego 27, 50-370 Wrocław, Poland \\ ${ }^{2}$ Institute of Computational Chemistry and Department of Chemistry, University of Girona, Campus de \\ Montilivi, 17071 Girona, Catalonia, Spain \\ ${ }^{3}$ Institute of Organic and Pharmaceutical Chemistry, National Hellenic Research Foundation, Vas. \\ Constantinou 48, GR-11635 Athens, Greece \\ ${ }^{4}$ Department of Physical Chemistry, Collegium Medicum, Nicolaus Copernicus University, Kurpińskiego 5, \\ 85-950 Bydgoszcz, Poland
}

(Received 1 July 2010; accepted 25 October 2010; published online 28 December 2010)

\begin{abstract}
In this study we report on the electronic and vibrational (hyper)polarizabilities of donor-acceptorsubstituted azobenzene. It is observed that both electronic and vibrational contributions to the electric dipole first hyperpolarizability of investigated photoactive molecule substantially depend on the conformation. The contributions to the nuclear relaxation first hyperpolarizability are found to be quite important in the case of two considered isomers ( $\mathrm{cis}$ and trans). Although the double-harmonic term is found to be the largest in terms of magnitude, it is shown that the total value of the nuclear relaxation contribution to vibrational first hyperpolarizability is a result of subtle interplay of higher-order contributions. As a part of the study, we also assess the performance of long-range-corrected density functional theory in determining vibrational contributions to electric dipole (hyper)polarizabilities. In most cases, the applied long-range-corrected exchange-correlation potentials amend the drawbacks of their conventional counterparts. (0) 2010 American Institute of Physics. [doi:10.1063/1.3516209]
\end{abstract}

\section{INTRODUCTION}

Among various molecular properties, molecular hyperpolarizabilities $(\beta, \gamma)$ have attracted special attention of scientific community for last three decades. As a result, new theoretical approaches and computational protocols have been proposed for evaluation of electronic and vibrational hyperpolarizabilities. ${ }^{1}$ The interest in accurate determining of $\beta$ and $\gamma$ is certainly because of potential applications of compounds with large hyperpolarizabilities in photonics. For example a plethora of organic and organometallic systems have been analyzed as potential optical switches. ${ }^{2,3}$ Among them, azobenzene derivatives (see Fig. 1) are still the subject of intense research. ${ }^{4,5}$ Azobenzene molecule is known to undergo photoisomerization from trans to cis conformer. The metastable cis form can return to trans isomer either thermally or after irradiation. ${ }^{6}$ The quite large value of the barrier to trans-cis isomerization, which can be modulated with donor and acceptor groups, ${ }^{6,7}$ makes the azobenzene derivatives candidate systems for their use as optical switches. A large difference between the nonlinear optical properties of both conformers will increase their potential as optical switches. In this paper we will analyze the dependence of the first hyperpolarizability on the trans-cis isomerization of the azobenzene derivative. Both electronic and vibrational

\footnotetext{
a) Author to whom correspondence should be addressed. Electronic mail: robert.zalesny@pwr.wroc.pl.

b) Permanent address: Department of Chemistry, Rice University, 6100 Main Street, Houston, Texas 77005-1892, USA.
}

contributions are computed in this work. The latter, although sometimes neglected, has an important weight in the value of the Pockels first hyperpolarizability.

Despite evident progress in the development of methods for evaluation vibrational (hyper)polarizabilities, ${ }^{8-20}$ their calculation for medium and large size molecules is much more computationally demanding than the electronic counterpart. The bottleneck of the calculation of the vibrational hyperpolarizabilities are the required high-order derivatives of energy and electronic properties with respect to the nuclear coordinates required for their evaluation. A significant step forward for the efficient evaluation of vibrational contributions to molecular hyperpolarizabilities was introduction of fieldinduced coordinates (FICs) method. ${ }^{21,22}$ In this technique, instead of 3N-6 normal coordinates one introduces a relatively small number of FICs to describe vibrational hyperpolarizabilities. It is now well settled that the vibrational hyperpolarizabilites can be even higher in terms of magnitude than their electronic counterpart. ${ }^{23}$ It has been shown only quite recently by Torrent-Sucarrat et al. ${ }^{16,24-26}$ and also by other authors ${ }^{27,28}$ that electron correlation effects have significant impact on the vibrational contributions to hyperpolarizabilities. In some cases, the $P^{\mathrm{vib}} / P^{\mathrm{e}}(P=\alpha, \beta, \gamma)$ ratio might depend crucially on the applied level of theory. ${ }^{29,30}$ Although an accurate determination of electronic and vibrational contributions to (hyper)polarizabilities of molecules in the gas phase is interesting in its own right, computational modeling of nonlinear vibrational spectroscopies is still very challenging task. ${ }^{14,31,32}$ Highly accurate post-Hartree-Fock 


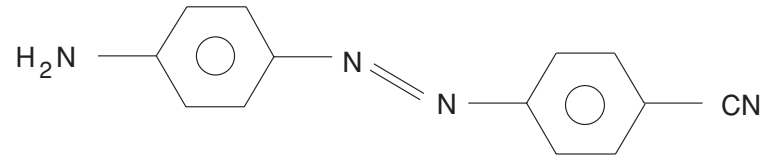

FIG. 1. Schematic representation of the investigated system.

(HF) theories are in most cases too computationally expensive to be efficiently applied. At first glance, the density functional theory (DFT) might appear to be a reliable tool in the field of vibrational spectroscopies. Unfortunately, still little is known about the performance of DFT in determining vibrational hyperpolarizabilities. ${ }^{17,33-35}$ Hence, one of the goals of this contribution is the assessment of recently proposed longrange-corrected (LRC) functionals in computing vibrational properties. As far as electronic contributions to nonlinear optical properties are concerned, these functionals were proven to be successful ${ }^{36-38}$ in curing an overshoot problem. Too large values of electronic (hyper)polarizabilities, reported at first for conjugated chain-like molecules, ${ }^{39-41}$ have their roots in self-interaction error. ${ }^{42}$ In the case of LRC potentials, the attenuation of Coulomb interactions is achieved by introduction of Hartree-Fock exchange at long range. Although the testing of exchange-correlation potentials is essential step in process of development of efficient methods to determine electric dipole (hyper)polarizabilities, the central point of the present study is the analysis of the change in the electronic and vibrational second-order nonlinear response of substituted azobenzene upon photoisomerization. Also of interest is the analysis of the weight of the anharmonicity on the nuclear relaxation first hyperpolarizabilities of D/A-substituted azobenzene. For this purpose we shall employ the FICs approach combined with the second-order Møller-Plesset perturbation theory to account for electron correlation effects.

\section{COMPUTATIONAL DETAILS}

In this section we will present only brief description of the methods used to determine electronic and vibrational (hyper)polarizabilities. The reader unfamiliar with the applied methods may refer to the vast literature on the subject. ${ }^{43}$ In the presence of an uniform electric field, the total energy of molecule can be expressed as a Taylor series:

$$
\begin{aligned}
E(F)= & E(0)-\mu_{i} F_{i}-\frac{1}{2 !} \alpha_{i j} F_{i} F_{j} \\
& -\frac{1}{3 !} \beta_{i j k} F_{i} F_{j} F_{k}-\frac{1}{4 !} \gamma_{i j k l} F_{i} F_{j} F_{k} F_{l} \cdots,
\end{aligned}
$$

where $E(0)$ denotes the field-free total energy and expansion coefficients $\mu, \alpha, \beta, \ldots$ are dipole moment, polarizability, and first- to $n$-th order hyperpolarizability, respectively. Equation (1) conforms to the so-called T-convention ${ }^{44}$ and shall be further used for calculations of electric dipole (hyper)polarizabilities. Within the Born-Oppenheimer approximation one may separate electronic and vibrational contributions to the property of interest $P(P=\alpha, \beta, \gamma, \ldots):^{43,45}$

$$
P=P^{\mathrm{e}}+P^{\mathrm{vib}} .
$$

Numerous approximations can be adopted to evaluate the two terms in the above equation. The details of the methods used to compute electronic and vibrational contributions in this work are given in the sections below.

\section{A. Electronic (hyper)polarizabilities}

In order to evaluate electronic contributions to (hyper)polarizabilities, we followed the procedure outlined by Kurtz and co-workers, ${ }^{46,47}$ and used Eq. (1) for numerical differentiation of the total energy of the molecular system with respect to the electric field. Many-body perturbation theory and coupled cluster calculations presented in this study were performed employing field-relaxed Hartree-Fock orbitals. In the case of orientationally invariant (hyper)polarizabilities: ${ }^{43}$

$$
\begin{aligned}
\bar{\alpha} & =\frac{1}{3} \sum_{i=x, y, z} \alpha_{i i}, \\
\bar{\beta} & =\sum_{i=x, y, z} \frac{\mu_{i} \beta_{i}}{|\mu|}, \\
& \text { where } \beta_{i}=\frac{1}{5} \sum_{j=x, y, z}\left(\beta_{i j j}+\beta_{j i j}+\beta_{j j i}\right),
\end{aligned}
$$

the constant value of the field amplitude (equal to 0.001 a.u.) was assumed. In the case of diagonal tensor elements the Romberg differentiation procedure was employed. ${ }^{48}$

\section{B. Vibrational (hyper)polarizabilities}

The vibrational contributions to a property $P\left(P^{\mathrm{vib}}\right)$ as defined in Eq. (2) may be further divided into nuclear relaxation $P^{\mathrm{NR}}$ and curvature $P^{\text {curv }}$ terms: ${ }^{43}$

$$
P^{\mathrm{vib}}=P^{\mathrm{NR}}+P^{\text {curv }} \text {. }
$$

The $P^{\mathrm{NR}}$ is usually larger than the curvature contribution. For this reason, the latter term is not computed here. Using the Bishop-Kirtman (BK) square bracket nomenclature the nuclear relaxation contributions to static (hyper)polarizabilities $\operatorname{read}^{49}$

$$
\begin{aligned}
& \alpha^{\mathrm{NR}}(0 ; 0)=\left[\mu^{2}\right]^{(0,0)}, \\
& \beta^{\mathrm{NR}}(0 ; 0,0)=[\mu \alpha]^{(0,0)}+\left[\mu^{3}\right]^{(1,0)}+\left[\mu^{3}\right]^{(0,1)},
\end{aligned}
$$

where for $[P]^{(n, m)}$ expressions, $n$ and $m$ are the order of electrical and mechanical anharmonicity, respectively. The terms inside square brackets denote energy/property derivatives. For instance the $[\mu \alpha]^{(0,0)}$ square bracket term is given by ${ }^{8-10}$

$$
[\mu \alpha]^{(0,0)}=\frac{1}{2} P_{a b c} \sum_{i=1}^{3 N-6}\left(\frac{\partial \alpha_{a b}}{\partial Q_{i}}\right)\left(\frac{\partial \mu_{c}}{\partial Q_{i}}\right) \omega_{i}^{-2},
$$

where $\omega_{i}$ are the vibrational frequencies, $a, b, c$ are the Cartesian coordinates $x, y, z$, and $P_{a b c}$ indicates a sum over all the perturbations of the indices $a b c$. For dc-Pockels first hyperpolarizability, within the infinite frequency approximation, only the lowest order term is sufficient to calculate the complete NR contribution: ${ }^{49}$

$$
\beta^{\mathrm{NR}}(-\omega ; \omega, 0)_{\omega \rightarrow \infty}=\frac{1}{3}[\mu \alpha]^{(0,0)} .
$$


TABLE I. Comparison of electronic and vibrational contributions to static first hyperpolarizability evaluated at various levels of theory.

\begin{tabular}{|c|c|c|c|c|}
\hline & $\bar{\beta}^{\mathrm{e}}$ & $\overline{[\mu \alpha]}^{(0,0)}$ & $\bar{\beta}^{\mathrm{e}}$ & $\overline{[\mu \alpha]}^{(0,0)}$ \\
\hline & \multicolumn{2}{|c|}{ trans isomer } & \multicolumn{2}{|c|}{ cis isomer } \\
\hline $\mathrm{HF} / 6-31 \mathrm{G}(\mathrm{d})$ & 1687 & 3843 & 494 & 8094 \\
\hline $\mathrm{HF} / 6-31+\mathrm{G}(\mathrm{d})$ & 1848 & 3891 & 565 & 8107 \\
\hline $\mathrm{MP} 2 / 6-31 \mathrm{G}(\mathrm{d})$ & 4112 & 4412 & 1058 & 8516 \\
\hline $\mathrm{MP} 2 / 6-31+\mathrm{G}(\mathrm{d})$ & 4414 & 4901 & 1115 & 9597 \\
\hline
\end{tabular}

The $[P]^{(0,0)}$ terms (see Tables I, III and IV) were computed using standard BK formulae, i.e., by summation over $3 N-6$ normal modes of vibration. In that event, the numerical differentiation of either the dipole moment or polarizability with respect to normal modes of vibration was performed to obtain relevant derivatives.

The vibrational contributions presented in Table $\mathrm{V}$ (see values in parentheses) were computed using the method proposed by Bishop et al., ${ }^{9}$ and later implemented by Luis et al. ${ }^{12}$ In this approach, the molecular geometry is first optimized in the presence of a static electric field while strictly maintaining the Eckart conditions. ${ }^{12}$ Then the difference in the static electronic properties due to the change in geometry induced by the field is expanded as a power series in the field. Each

TABLE II. Purely electronic contributions to first-order hyperpolarizability calculated for the geometry optimized at the MP2/6-31G(d) level of theory.

\begin{tabular}{|c|c|c|c|c|}
\hline & $\beta_{z z z}^{\mathrm{e}}$ & $\bar{\beta}^{\mathrm{e}}$ & $\beta_{z z z}^{\mathrm{e}}$ & $\bar{\beta}^{\mathrm{e}}$ \\
\hline & \multicolumn{2}{|c|}{ trans isomer } & \multicolumn{2}{|c|}{ cis isomer } \\
\hline B3LYP/PC1 & -6589 & 4007 & -2443 & 1490 \\
\hline B3LYP/PC2 & -6834 & 4159 & -2254 & 1525 \\
\hline B3LYP/6-31G(d) & -6367 & 3845 & -2340 & 1426 \\
\hline CAM-B3LYP/PC1 & -5626 & 3404 & -1610 & 1081 \\
\hline CAM-B3LYP/PC2 & -5814 & 3520 & -1642 & 1113 \\
\hline CAM-B3LYP/6-31G(d) & -5337 & 3229 & -1525 & 1030 \\
\hline BLYP/PC1 & -6787 & 4142 & -2697 & 1851 \\
\hline BLYP/PC2 & -7164 & 4372 & -2783 & 1919 \\
\hline BLYP/6-31G(d) & -6651 & 4024 & -2903 & 1781 \\
\hline LC-BLYP/PC1 & -5182 & 3134 & -1422 & 961 \\
\hline LC-BLYP/aug-PC1 & -5266 & 3194 & -1416 & 967 \\
\hline LC-BLYP/PC2 & -5402 & 3274 & -1468 & 1005 \\
\hline LC-BLYP/6-31G(d) & -4913 & 2959 & -1504 & 913 \\
\hline $\mathrm{BOP} / \mathrm{PC} 1$ & -6832 & 4170 & -2705 & 1854 \\
\hline $\mathrm{BOP} / \mathrm{PC} 2$ & -7189 & 4387 & -2780 & 1917 \\
\hline $\mathrm{BOP} / 6-31 \mathrm{G}(\mathrm{d})$ & -6641 & 4054 & -2600 & 1786 \\
\hline LC-BOP/PC1 & -5169 & 3126 & -1415 & 957 \\
\hline LC-BOP/PC2 & -5382 & 3252 & -1641 & 995 \\
\hline LC-BOP/6-31G(d) & -4891 & 2958 & -1339 & 911 \\
\hline $\mathrm{HF} / \mathrm{PC} 1$ & -3752 & 2262 & -945 & 573 \\
\hline $\mathrm{HF} / \mathrm{PC} 2$ & -3858 & 2330 & -872 & 589 \\
\hline $\mathrm{HF} / 6-31 \mathrm{G}(\mathrm{d})$ & -3530 & 2123 & -889 & 539 \\
\hline MP2/cc-pVDZ & -6668 & 4136 & -1537 & 1038 \\
\hline MP2/cc-pVTZ & -6998 & 4346 & -1599 & 1090 \\
\hline $\mathrm{MP} 2 / 6-31 \mathrm{G}(\mathrm{d})$ & -6654 & 4112 & -1565 & 1058 \\
\hline MP2/aug-cc-pVDZ & -7274 & 4503 & -1659 & 1140 \\
\hline $\operatorname{CCSD}(\mathrm{T}) / 6-31 \mathrm{G}(\mathrm{d})$ & -6176 & 3881 & -1542 & 1074 \\
\hline
\end{tabular}

term in the expansion yields the sum of a static electronic (hyper)polarizability plus a nuclear relaxation vibrational term. For example, the change of the dipole moment is given by $^{9}$

$$
\Delta \mu_{i}\left(F, R_{F}\right)=a_{1, i j} F_{j}+\frac{1}{2} b_{1, i j k} F_{j} F_{k}+\cdots,
$$

where

$$
a_{1, i j}=\alpha_{i j}^{\mathrm{e}}(0 ; 0)+\alpha_{i j}^{\mathrm{NR}}(0 ; 0),
$$

$$
b_{1, i j k}=\beta_{i j k}^{\mathrm{e}}(0 ; 0,0)+\beta_{i j k}^{\mathrm{NR}}(0 ; 0,0) .
$$

The argument $R_{F}$ refers to the field-relaxed geometry of a molecule.

In order to evaluate explicitly the first-order electrical and mechanical contributions to the vibrational firsthyperpolarizability (see middle columns of Table V), an approach based on the FICs, which are linear combinations of field-free normal coordinates associated with the change in equilibrium geometry induced by a static electric field, has been employed. ${ }^{21}$ The displacement of the $i$-th field-free normal coordinate at the field-relaxed geometry is given by ${ }^{21}$

$$
\begin{aligned}
Q_{i}^{F}\left(F_{x}, F_{y}, F_{z}\right)= & -\sum_{a}^{x, y, z} q_{1}^{i, a} F_{a} \\
& -\sum_{a, b}^{x, y, z}\left[q_{2}^{i, a b}-\sum_{j=1}^{3 N-6} \frac{a_{21}^{i j, a}}{a_{20}^{i i}} q_{1}^{i, b}\right. \\
& \left.+\sum_{j, k=1}^{3 N-6} \frac{3 a_{30}^{i j k}}{2 a_{20}^{i i}} q_{1}^{j, a} q_{1}^{k, b}\right] F_{a} F_{b}+\cdots,
\end{aligned}
$$

where

$$
\begin{aligned}
& a_{n m}^{i j \cdots a b \cdots}=\frac{1}{n ! m !} \\
& \times\left(\frac{\partial^{(n+m)} V\left(Q_{1}, \ldots, Q_{3 N-6}, F_{x}, F_{y}, F_{z}\right)}{\partial Q_{i} \partial Q_{j} \cdots \partial F_{a} \partial F_{b} \cdots}\right)_{Q=0, F=0}, \\
& q_{1}^{i, a}=\frac{a_{11}^{i, a}}{2 a_{20}^{i i},} \quad q_{2}^{i, a b}=\frac{a_{12}^{i, a b}}{2 a_{20}^{i i}} .
\end{aligned}
$$

The indices $i, j, k$ refer to normal coordinates whereas $a, b, c$ label the Cartesian directions along molecular axes. $a_{n m}$ involves the $n$-th and $m$-th derivatives of the potential energy $V(Q, F)$ with respect to normal coordinates and field components, respectively. The first-order FICs are sufficient to compute static nuclear relaxation contribution to the linear polarizability and first hyperpolarizability: ${ }^{21}$

$$
\chi_{1}^{a}=-\sum_{i=1}^{3 N-6} q_{1}^{i, a} Q_{i} .
$$

Depending on the nonlinear optical property of interest, a small set of FICs, independent of the number of atoms in the 
TABLE III. Nuclear relaxation and electronic contributions to molecular first hyperpolarizability calculated using the 6-31G(d) basis set. All values are given in atomic units.

\begin{tabular}{|c|c|c|c|c|c|c|c|c|}
\hline & $\bar{\alpha}^{\mathrm{NR}}(0 ; 0)$ & $\bar{\alpha}^{\mathrm{e}}$ & $\bar{\beta}^{\mathrm{NR}}(-\omega ; \omega, 0)$ & $\frac{1}{3} \bar{\beta}^{\mathrm{e}}$ & $\bar{\alpha}^{\mathrm{NR}}(0 ; 0)$ & $\bar{\alpha}^{\mathrm{e}}$ & $\bar{\beta}^{\mathrm{NR}}(-\omega ; \omega, 0)$ & $\frac{1}{3} \bar{\beta}^{\mathrm{e}}$ \\
\hline & \multicolumn{4}{|c|}{ trans isomer } & \multicolumn{4}{|c|}{ cis isomer } \\
\hline BLYP & 73.6 & 238.4 & 2433 & 1364 & 91.4 & 195.7 & 4139 & 643 \\
\hline BOP & 60.9 & 239.3 & 2122 & 1393 & 94.8 & 195.8 & 4381 & 658 \\
\hline B3LYP & 58.7 & 217.1 & 2314 & 1357 & 87.3 & 178.5 & 3799 & 552 \\
\hline LC-BLYP & 55.8 & 188.1 & 1860 & 963 & 81.0 & 161.2 & 3519 & 333 \\
\hline LC-BOP & 57.1 & 188.4 & 1890 & 965 & 79.3 & 161.3 & 3500 & 330 \\
\hline CAM-B3LYP & 55.9 & 195.6 & 2082 & 1092 & 81.5 & 165.0 & 3415 & 392 \\
\hline $\mathrm{HF}$ & 46.6 & 172.3 & 1279 & 562 & 66.1 & 150.2 & 2697 & 165 \\
\hline MP2 & 49.2 & 188.4 & 1471 & 1371 & 62.7 & 157.8 & 2839 & 353 \\
\hline
\end{tabular}

molecule, may be employed to calculate the total NR contribution instead of using all 3N-6 normal coordinates. For example, from the three first-order FICs (one for each Cartesian direction) one can compute the nuclear relaxation contributions to the static polarizability and first hyperpolarizability, as

$$
\begin{aligned}
& \alpha_{a b}^{\mathrm{NR}}(0 ; 0)=\frac{1}{2} \sum P_{a b} \frac{\partial \mu_{a}}{\partial \chi_{1}^{b}} \frac{\partial \chi_{1}^{F}}{\partial F_{b}}=\left[\mu^{2}\right]^{(0,0)}, \\
& \beta_{a b c}^{\mathrm{NR}}(0 ; 0,0)= \sum P_{a b c}\left[\frac{1}{2} \frac{\partial \alpha_{a b}}{\partial \chi_{1}^{c}} \frac{\partial \chi_{1}^{F}}{\partial F_{c}}\right. \\
&+\frac{\partial^{2} \mu_{a}}{\partial \chi_{1}^{b} \partial \chi_{1}^{c}} \frac{\partial \chi_{1}^{F}}{\partial F_{b}} \frac{\partial \chi_{1}^{F}}{\partial F_{c}} \\
&\left.-\frac{1}{6} \frac{\partial^{3} V}{\partial \chi_{1}^{a} \partial \chi_{1}^{b} \partial \chi_{1}^{c}} \frac{\partial \chi_{1}^{F}}{\partial F_{a}} \frac{\partial \chi_{1}^{F}}{\partial F_{b}} \frac{\partial \chi_{1}^{F}}{\partial F_{c}}\right] \\
&= {[\mu \alpha]^{(0,0)}+\left[\mu^{3}\right]^{(1,0)}+\left[\mu^{3}\right]^{(0,1)}, }
\end{aligned}
$$

where $\sum P_{a b \ldots}$ indicates the sum over all permutations of the indices $a, b, \ldots$. More details about this method can be found elsewhere. $^{21}$

Derivatives with respect to the FIC coordinate were obtained numerically, at the field-free optimum geometry, by applying a number of steps along the FIC vector.

Computations with the aid of post-Hartree-Fock methods were done within the frozen-core approximation. Electronic and vibrational contributions to $\alpha$ and $\beta$ were determined using the GAMESS US, ${ }^{50}$ the GAUSSIAN $09,{ }^{51}$ and the modified version of the DALTON program. ${ }^{52}$

\section{RESULTS AND DISCUSSION}

As mentioned in Introduction, the investigated system undergoes a significant structural transformation upon elec-

TABLE IV. $[\mu \beta]^{(0,0)}$ contribution to the vibrational second-order hyperpolarizability ( $\gamma_{z z z z}$, given in a.u.) calculated at various levels of theory.

\begin{tabular}{lccc}
\hline \hline & B3LYP/6-31G(d) & CAM-B3LYP/6-31G(d) & MP2/6-31G(d) \\
\hline trans isomer & $143 \times 10^{3}$ & $483 \times 10^{3}$ & $647 \times 10^{3}$ \\
cis isomer & $126 \times 10^{3}$ & $403 \times 10^{3}$ & $542 \times 10^{3}$ \\
\hline \hline
\end{tabular}

tronic excitation. The geometries of the molecule optimized at the MP2/6-31G(d) level of theory are presented in electronic supplementary information (ESI). ${ }^{53}$ The average difference in bond lengths presented in ESI in Fig. 1 is not significant and is equal to $0.012 \AA$. Thus, the main structural difference upon trans-cis transformation is the change of dihedral angles involving $N=N$ moiety. In a result, the electronic dipole moment decreases from 7.4 to 4.1 D. The changes are also reflected in the values of average electronic and vibrational static first hyperpolarizability (see Table I). The absolute values of $\Delta \bar{\beta}_{\text {trans } \rightarrow \text { cis }}^{\mathrm{e}}$ (and $\Delta \bar{\beta}_{\text {trans } \rightarrow \text { cis }}^{\mathrm{V}}$ ) might suggest that trans $\rightarrow$ cis isomerization leads to large changes in both vibrational and electronic structure. The reliability of the double-harmonic oscillator model, used for computing part of the data from Table I, in determining vibrational counterpart of molecular hyperpolarizabilities shall be discussed further. As it has already been shown, the ratio $\beta^{\mathrm{v}} / \beta^{\mathrm{e}}$ might crucially depend on the level of theoretical approximation. ${ }^{25,30}$ Indeed, the values of electronic and vibrational hyperpolarizabilities seem to confirm previous findings. In the case of both conformers, the inclusion of electron correlation effects diminishes the $\beta^{\mathrm{v}} / \beta^{\mathrm{e}}$ ratio approximately by a factor of 2 . This is because the HF approximation significantly underestimates the values of $\beta^{\mathrm{e}}$ in comparison with the MP2 results. The latter method is not always a good reference point; however, as it will be shown later, it provides very reliable estimate of electronic contributions to $\beta$ for investigated molecules. The other issue is the choice of the basis set. The $6-31+\mathrm{G}(\mathrm{d})$ basis set was found by Torrent-Succarrat et al. to provide the results of "semiquantitative accuracy." 25 The difference in vibrational first hyperpolarizability computed in this study using the two basis sets at the MP2 level of theory is not large and does not exceed $15 \%$. Hence, we decided to employ the smaller 6-31G(d) basis set for computing the vibrational properties.

In order to elucidate the effect of the level of theory (including the influence of basis set) on the electronic contributions to $\beta$, the extensive computations were performed using both $a b$ initio methods as well as several exchangecorrelation potentials commonly used within the density functional theory framework. The results are presented in Table II. Contrary to the data discussed above, all results of computations from Table II correspond to fixed geometries optimized at the MP2/6-31G(d) level of theory. This allows to compare directly the values of purely electronic 
TABLE V. Harmonic and anharmonic contributions to the vibrational first-order hyperpolarizability calculated at the MP2/6-31G(d) level of theory. The values calculated using the HF/B3LYP/CAM-B3LYP methods with the 6-31G(d) basis set are given in parentheses. All values are given in atomic units and should be multiplied by $10^{3}$.

\begin{tabular}{lcccc}
\hline \hline & {$[\mu \alpha]_{z z z}^{(0,0)}$} & {$\left[\mu^{3}\right]_{z z z}^{(1,0)}$} & {$\left[\mu^{3}\right]_{z z z}^{(0,1)}$} & $\beta_{z z z}^{\mathrm{NR}}$ \\
\hline trans isomer & $-5.7(-5.2 /-10.2 /-8.8)$ & 0.8 & -2.1 & $-7.1(-8.2 /-21.8 /-18.8)$ \\
cis isomer & $-12.6(-13.7 /-18.2 /-17.2)$ & -2.1 & -4.2 & $-18.9(-21.1 /-22.9 /-26.9)$ \\
\hline \hline
\end{tabular}

(hyper)polarizabilities computed using various approaches. Even though the 6-31G(d) basis set is not easily comparable to the cc-pVXZ basis sets, the values of $\beta$ determined using the MP2 method show an acceptable convergence with respect to the basis set size. As expected, augmentation of the cc-pVDZ basis set with diffuse functions further increases the values of first hyperpolarizability. It should be underlined that in the case of both conformers the differences in electronic contributions to $\beta$ arising from the basis set used in calculations are not large and does not exceed $10 \%$. Similarly to other organic compounds, the MP2 method tends to overestimate the absolute values of hyperpolarizabilities as compared to the $\operatorname{CCSD}(\mathrm{T})$ approach. Likewise, the differences are not significant. Based on the ab initio data presented in both tables, the MP2 method combined with 6-31G(d) basis set was selected as reliable and quite accurate level of approximation to study vibrational properties which are of primary interest for this study. Turning toward the results presented in Table II, let us comment on the performance of density functional theory in determining electronic contributions to $\beta$. Besides the Pople's 6-31G(d) basis set, we have also computed electronic hyperpolarizabilities using Jensen's pc- $n$ basis sets as they are specifically designed for HF and DFT calculations. ${ }^{54,55}$ The pc-1 and pc-2 basis set in terms of size correspond to Dunning's correlation consistent cc-pVDZ and cc-pVTZ basis sets. The first conclusion is that all applied exchange-correlation potentials improve upon Hartree-Fock approximation. The latter method leads to significantly underestimated values of $\beta$ in comparison with the MP2 or the $\operatorname{CCSD}(\mathrm{T})$ treatments. Secondly, one finds that the longrange corrected functionals do not improve systematically upon their conventional counterparts. It is quite striking that in the case of trans conformer they worsen the results both for diagonal and average hyperpolarizability. On the contrary, for cis isomer they provide smaller values of hyperpolarizability which are in better agreement with the MP2/CCSD(T) data.

In order to make an assessment of the density functional theory in determining nonlinear optical response, arising from nuclear motions, the nuclear relaxation contributions to $\alpha$ and $\beta$ were determined for three commonly used exchangecorrelation potentials as well as for their long-range-corrected counterparts. The results of computations are presented in Table III. In each case, the equilibrium geometry and the values of electronic and vibrational properties were determined at the same level of theory. First, the electronic and the NR contributions to polarizability are overestimated by conventional functionals and the LRC potentials partially cure the overshoot problem. This is true for both isomers. It is important to remark that for $\alpha^{\mathrm{NR}} \mathrm{HF}$ gives more accurate values than any of the studied DFT functionals. As far as the nuclear relaxation first hyperpolarizability is concerned, the general trends are similar to $\alpha^{\mathrm{NR}}$. The nuclear relaxation contributions to $\beta$ for dc-Pockels process are predicted significantly better by the LRC functionals than by their conventional counterparts. Again, conventional DFT functionals overshoot the MP2 result for $\beta^{\mathrm{NR}}$ whereas the LRC functionals partially cure this problem. And also for $\beta^{\mathrm{NR}}$ no functional improves the results of HF, which gives quite accurate values.

Table III shows that the nuclear relaxation contribution to Pockels hyperpolarizability for cis isomer is about two times larger than their counterpart for trans isomer. This vibrational contribution is given by the square bracket $\overline{[\mu \alpha}^{(0,0)}$ term, which involves summation over derivatives of electric dipole moment and polarizability with respect to nuclear displacements and vibrational frequencies [see Eq. (8)]. In ESI (cf. Table II), the contributions from individual normal modes of vibration to first hyperpolarizability for the two isomers are presented. ${ }^{53}$ For the cis conformer the lowest frequency mode is found to determine solely vibrational contribution to $\beta$. Contrary, in the case of trans form the largest contribution comes from mode denoted as 26 . A close look at dipole moment and polarizability derivatives with respect to given normal mode reveals that $\partial \mu_{z}^{\text {trans }} / \partial \mathbf{Q}_{26} \gg \partial \mu_{z}^{\text {cis }} / \partial \mathbf{Q}_{1}$ but $\partial \alpha_{z z}^{\text {trans }} / \partial \mathbf{Q}_{26} \approx \partial \alpha_{z z}^{\text {cis }} / \partial \mathbf{Q}_{1}$. On the other hand $\omega_{26}^{\text {trans }}$ is about 20 times larger than $\omega_{1}^{c i s}$. Thus, it is the vibrational frequency of the key normal mode that determines the nuclear relaxation contribution which causes that vibrational first hyperpolarizability is larger for cis rather than for trans conformer.

The working formulae for $\beta_{\mathrm{dc}-\mathrm{P}}^{\mathrm{NR}}$ involve summation over derivatives of electric dipole moment and polarizability with respect to nuclear displacements. Since the values of electronic first hyperpolarizability seem to be much more sensitive to the XC potential used in calculations than the polarizability is, the nuclear derivatives of $\beta$ should depend to a larger extent on the applied exchange-correlation potential. Indeed, the results of calculations presented in Table IV indicate significant discrepancy in $[\mu \beta]^{(0,0)}$ values obtained using conventional B3LYP functional and the MP2 method. The computed term makes a contribution to vibrational second hyperpolarizability. Contrary to the purely electronic hyperpolarizabilities of extended $\pi$-conjugated systems, ${ }^{40}$ no overshoot is observed here, i.e., the values predicted by the B3LYP potential are significantly underestimated. The long-range correction improves the poor performance of the B3LYP functional. The ratio of the $[\mu \beta]^{(0,0)}$ values for both isomers is the same for the CAM-B3LYP functional and the MP2 method.

The values of various contributions to the vibrational first hyperpolarizability presented in Table $\mathrm{V}$ were performed for equilibrium geometries oriented in such a way that in each case the dipole moment vector was parallel to 
Cartesian $z$ axis. This geometry dependence of the results could increase the discrepancies of the different theoretical methods. Unfortunately, due to the complexity of the computational protocol used to determine the vibrational properties, we have not attempted to evaluate the full hyperpolarizability tensor. Nevertheless, some valuable conclusions regarding theoretical methods can be drawn based on the data presented in Table V. First, despite the small differences in geometries and in the orientation in Cartesian coordinate system, the nuclear relaxation contributions to $\beta$ are predicted by both applied functionals to be similar in terms of magnitude. Secondly, the sum of first-order corrections (i.e., $\beta_{z z z}^{\mathrm{NR}}-[\mu \alpha]_{z z z}^{(0,0)}$ ) is negative at all applied levels of theory. Thirdly, also for the anharmonic NR contribution to $\beta$ the HF results are far more similar to the MP2, which based on Table II data we use as reference, than the two DFT functionals studied. However, the contribution through first order in electrical anharmonicity might be either positive (trans isomer) or negative (cis isomer). In the considered case, the lowest order of the BK perturbation theory seem to be only crude approximation to the vibrational static first hyperpolarizability. As pointed previously, the calculation of the higher order anharmonic terms for the full tensor have a high computational cost. However, at this point it is important to remark that first order $\left[\mu^{3}\right]$ term has no role in the calculation of nuclear relaxation contribution to Pockels first hyperpolarizability within the infinite frequency approximation [see Eq. (9)].

\section{SUMMARY}

We have considered both electronic and vibrational (hyper)polarizabilities of donor-acceptor-substituted azobenzene. Nowadays, azobenzene derivatives are widely used as building blocks of hybrid systems of high nonlinear optical activity. As a part of a model study, we have assessed the performance of density functional theory in determining electric dipole (hyper)polarizabilities. In particular, recently proposed long-range-corrected exchange-correlation potentials were considered. As it turned out, in many cases they partially amend the drawbacks of their conventional counterparts, as for instance being successful in determining vibrational first and second hyperpolarizability. We have also noted that of the two contributions to first hyperpolarizability, namely electronic and vibrational, the former is influenced to a much larger extent by electron correlation effects. As a result, the Hartree-Fock method does not seem to be fortunate choice for determining relations between vibrational and electronic hyperpolarizabilities. Nevertheless, in this particular case, HF seems to be a good approach to calculate the nuclear-relaxation contribution values.

The nonlinear optical response of D/A-substituted azobenzene crucially depends on the conformation. Orientationally invariant electronic first hyperpolarizability is four times larger for trans than for cis isomer. The vibrational contributions behave differently, i.e., they are approximately two times larger for the latter isomer. We have also determined, with the inclusion of electron correlation effects by means of the MP2 method, the anharmonic contributions to vibrational first hyperpolarizability through the first order in electrical and mechanical anharmonicity. We noticed that the value of the so-called nuclear relaxation contribution to vibrational first hyperpolarizability is a subtle balance between the three contributions among which the double-harmonic term is found to be the largest in terms of magnitude. However, the values of individual components, and particularly their signs, are warning not to neglect the first-order anharmonic corrections as they might be quite important and strongly depend on the molecular geometry.

\section{ACKNOWLEDGMENTS}

This work was supported by computational grants from Wroclaw Center for Networking and Supercomputing (WCSS) and ACK Cyfronet. One of the authors (R.Z.) is the recipient of the fellowship cofinanced by European Union within European Social Fund. R.Z. would also like to acknowledge support from a grant from Iceland, Liechtenstein, and Norway through the EEA Financial MechanismScholarship and Training Fund. The support from the European Union (MTKD-CT-2006-042488), the Barcelona Supercomputing Center, and grants to access the TeraGrid facility (TG-CHE080063N) and the DEISA infrastructure are gratefully acknowledged. R.Z. and W.B. are grateful for financial support from Wroclaw University of Technology.

${ }^{1}$ P. Sałek, O. Vahtras, T. Helgaker, and H. Ågren, J. Chem. Phys. 117, 9630 (2002).

${ }^{2}$ B. J. Coe, in Nonlinear Optical Properties of Matter, edited by M. G. Papadopoulos, J. Leszczynski, and A. Sadlej (Springer-Verlag, Berlin, 2006).

${ }^{3}$ K. A. Green, M. P. Cifuentes, T. C. Corkery, M. Samoc, and M. G. Humphrey, Angew. Chem., Int. Ed. 48, 7867 (2009).

${ }^{4}$ S. Kawata and Y. Kawata, Chem. Rev. 100, 1777 (2000).

${ }^{5}$ S. Wagner, F. Leyssner, C. Koerdel, S. Zarwell, R. Schmidt, M. Weinelt, K. Rueck-Braun, M. Wolf, and P. Tegeder, Phys. Chem. Chem. Phys. 11, 6242 (2009).

${ }^{6}$ N. Nishimura, S. Kosako, and Y. Sueishi, Bull. Chem. Soc. Jpn. 57, 1617 (1984).

${ }^{7}$ C. R. Crecca and A. E. Roitberg, J. Phys. Chem. A 110, 8188 (2006).

${ }^{8}$ D. M. Bishop and B. Kirtman, J. Chem. Phys. 95, 2646 (1991).

${ }^{9}$ D. M. Bishop, M. Hasan, and B. Kirtman, J. Chem. Phys. 103, 4157 (1995).

${ }^{10}$ B. Kirtman, J. M. Luis, and D. M. Bishop, J. Chem. Phys. 108, 10008 (1998).

${ }^{11}$ J. M. L. J. Martí, M. Duran, J. L. Andrés, and B. Kirtman, J. Chem. Phys. 108, 4123 (1998).

${ }^{12}$ J. M. Luis, M. Duran, J. L. Andrés, B. Champagne, and B. Kirtman, J. Chem. Phys. 111, 875 (1999).

${ }^{13}$ O. Quinet, B. Kirtman, and B. Champagne, J. Chem. Phys. 118, 505 (2003).

${ }^{14}$ R. Zaleśny, W. Bartkowiak, and B. Champagne, Chem. Phys. Lett. 380, 549 (2003).

${ }^{15}$ D. M. Bishop, J. M. Luis, and B. Kirtman, J. Chem. Phys. 116, 9729 (2002).

${ }^{16}$ M. Torrent-Sucarrat, J. M. Luis, and B. Kirtman, J. Chem. Phys. 122, 204108 (2005).

${ }^{17}$ A. J. Thorvaldsen, K. Ruud, and M. Jaszuński, J. Phys. Chem. A 112, 11942 (2008).

${ }^{18}$ J. Kongsted and O. Christiansen, J. Chem. Phys. 127, 154315 (2007).

${ }^{19}$ O. Christiansen, Phys. Chem. Chem. Phys. 9, 2942 (2007).

${ }^{20}$ P. Seidler, J. Kongsted, and O. Christiansen, J. Phys. Chem. A 111, 11205 (2007)

${ }^{21}$ J. M. Luis, M. Duran, B. Champagne, and B. Kirtman, J. Chem. Phys. 113, 5203 (2000).

${ }^{22}$ J. M. Luis, M. Duran, and B. Kirtman, J. Chem. Phys. 115, 4473 (2001).

${ }^{23}$ D. P. Shelton and L. Ulivi, J. Chem. Phys. 89, 149 (1988). 
${ }^{24}$ M. Torrent-Sucarrat, M. Sola, M. Duran, J. M. Luis, and B. Kirtman, J. Chem. Phys. 116, 5363 (2002).

${ }^{25}$ M. Torrent-Sucarrat, M. Sola, M. Duran, J. M. Luis, and B. Kirtman, J. Chem. Phys. 118, 711 (2003).

${ }^{26}$ M. Torrent-Sucarrat, M. Sola, M. Duran, J. M. Luis, and B. Kirtman, J. Chem. Phys. 120, 6346 (2004).

${ }^{27}$ W. Bartkowiak, W. Niewodniczański, T. Misiaszek, and R. Zaleśny, Chem. Phys. Lett. 411, 8 (2005).

${ }^{28}$ R. Zaleśny, W. Bartkowiak, P. Toman, and J. Leszczynski, Chem. Phys. 337, 77 (2007).

${ }^{29}$ B. Champagne, T. Legrand, E. A. Perpète, O. Quinet, and J. M. André, Collect. Czech. Chem. Commun. 63, 1295 (1998).

${ }^{30}$ R. Zaleśny, M. G. Papadopoulos, W. Bartkowiak, and A. Kaczmarek, J. Chem. Phys. 129, 134310 (2008).

${ }^{31}$ O. Quinet, Int. J. Quantum Chem. 104, 727 (2005).

${ }^{32}$ E. Botek and B. Champagne, Appl. Phys. B 74, 627 (2002).

${ }^{33}$ E. A. Perpète, B. Champagne, and B. Kirtman, Phys. Rev. B 61, 13137 (2000).

${ }^{34}$ M. R. Pederson, T. Baruah, P. B. Allen, and C. Schmidt, J. Chem. Theory Comput. 1, 590 (2005).

${ }^{35}$ R. Zaleśny, G. Wójcik, I. Mossakowska, W. Bartkowiak, A. Avramopoulos, and M. G. Papadopoulos, J. Mol. Struct.: THEOCHEM 907, 46 (2009).

${ }^{36}$ B. Kirtman, S. Bonness, A. Ramirez-Solis, B. Champagne, H. Matsumoto, and H. Sekino, J. Chem. Phys. 128, 114108 (2008).

${ }^{37}$ O. Loboda, R. Zaleśny, A. Avramopoulos, J. M. Luis, B. Kirtman, N. Tagmatarchis, H. Reis, and M. G. Papadopoulos, J. Phys. Chem. A 113, 1159 (2009).

${ }^{38}$ D. Jacquemin, E. A. Perpète, M. Medved, G. Scalmani, M. J. Frisch, R. Kobayashi, and C. Adamo, J. Chem. Phys. 126, 191108 (2007).

${ }^{39}$ B. Champagne, E. A. Perpète, S. J. A. van Gisbergen, E. J. Baerends, J. G. Snijders, C. Soubra-Ghaoui, K. A. Robins, and B. Kirtman, J. Chem. Phys. 109, 10489 (1998b).
${ }^{40}$ B. Champagne, E. A. Perpète, D. Jacquemin, S. J. A. van Gisbergen, E. J. Baerends, C. Soubra-Ghaoui, K. A. Robins, and B. Kirtman, J. Phys. Chem. A 104, 4755 (2000).

${ }^{41}$ S. J. A. van Gisbergen, P. R. T. Schipper, O. V. Gritsenko, E. J. Baerends, J. G. Snijders, B. Champagne, and B. Kirtman, Phys. Rev. Lett. 83, 694 (1999).

${ }^{42}$ A. Dreuw and M. Head-Gordon, Chem. Rev. 105, 4009 (2005).

${ }^{43}$ D. Bishop and P. Norman, Handbook of Advanced Electronic and Photonic Materials and Devices, Vol. 9, edited by H. S. Nalwa (Academic, San Diego, 2001).

${ }^{44}$ A. Willets, J. Rice, D. Burland, and D. Shelton, J. Chem. Phys. 97, 7590 (1992).

${ }^{45}$ B. Champagne and B. Kirtman, Handbook of Advanced Electronic and Photonic Materials and Devices, Vol. 9, edited by H. S. Nalwa (Academic, San Diego, 2001).

${ }^{46}$ H. Kurtz, J. Stewart, and K. Dieter, J. Comput. Chem. 11, 82 (1990).

${ }^{47}$ H. A. Kurtz and D. S. Dudis, Reviews in Computational Chemistry, Vol. 12, edited by K. B. Lipkowitz and D. B. Boyd (Wiley-VCH, Weinheim, 1998), pp. 241-279.

${ }^{48}$ M. Medved, M. Stachová, D. Jacquemin, J.-M. André, and E. A. Perpète, J. Mol. Struct.: THEOCHEM 847, 39 (2007).

${ }^{49}$ B. Kirtman, B. Champagne, and J. M. Luis, J. Comput. Chem. 21, 1572 (2000).

${ }^{50}$ M. W. Schmidt, K. K. Baldridge, J. A. Boatz, S. T. Elbert, M. S. Gordon, J. H. Jensen, S. Koseki, N. Matsunaga, K. A. Nguyen, S. Su, T. L. Windus, M. Dupuis, and J. A. Montgomery, J. Comput. Chem. 14, 1347 (1993).

${ }^{51}$ M. J. Frisch, G. W. Trucks, H. B. Schlegel et al., Gaussian 09 Revision A.1, Gaussian Inc., Wallingford, CT, 2009.

${ }^{52}$ DALTON, a molecular electronic structure program, Release 2.0 (2005), see http://www.kjemi.uio.no/software/dalton/dalton.html.

${ }^{53}$ See supplementary material at http://dx.doi.org/10.1063/1.3516209 for vibrational analysis.

${ }^{54}$ F. Jensen, J. Chem. Phys. 115, 9113 (2001).

${ }^{55}$ F. Jensen, J. Chem. Phys. 116, 7372 (2002). 